\title{
FAST METHOD TO INCLUDE PARASITIC COUPLING IN PLANAR MICROWAVE CIRCUITS CALCULATIONS
}

\author{
B. L. A. Van Thielen ${ }^{1}$ and G.A. E. Vandenbosch. ${ }^{1}$ \\ ${ }^{1}$ vthielen@esat.kuleuven.ac.be and vdbosch@esat.kuleuven.ac.be \\ ESAT/TELEMIC \\ Katholieke Universiteit Leuven \\ Kasteelpark 10, B-3000 Leuven, Belgium
}

\begin{abstract}
As modern circuits become smaller and the frequencies that they work at become higher, inevitably, parasitic coupling within the circuits starts to influence the behavior of the circuit more and more. Therefore it is necessary to include the influence of mutual coupling in the circuit simulators that are used to design the circuit.

In this paper the circuit is divided into three classes of building blocks: transmission lines, small discontinuities (components) and meshed structures (solved using classical MoM). We will describe software modules that can calculate the couplings between these classes. The three modules are compatible with each other because they use the same parameters (incoming and outgoing waves at the ports, incident fields and radiating currents). They can be easily combined with a normal circuit simulation engine to include mutual coupling.
\end{abstract}

\section{COUPLING BETWEEN TRANSMISSION LINES}

This section explains how coupling between lines can be calculated much faster by using the eigenmodes (traveling waves) that exist on matched transmission lines. Only first order coupling is calculated: the radiation effect of the induced currents is neglected. The line - line couplings are calculated for each combination of two lines separately, while discarding all other objects in the circuit. The theory that is used to calculate line coupling is described in detail in [1]. It will be briefly described here.

We suppose that only one of the two lines is fed (the source line) and we want to calculate the outgoing transmission line waves on the other line (the observation line). We also suppose that both lines are terminated in impedances that are equal to their characteristic impedance. This problem has to be solved for an arbitrary configuration of the two lines. One classical way to do this is to use a Method of Moments. The calculations can be speeded up a lot if we assume that the current on the source line is not influenced by the proximity of the observation line. This means however that the lines can not be coupled to tightly (maximum about $-7 \mathrm{~dB}$ ). If the coupling is tighter than this then the results can still be improved by applying the method again. This time the observation line is regarded as the source line, which is carrying the previously calculated current. Extra iteration steps like this can be applied until sufficient accuracy is reached.

Because we assume that the source line is not influenced by the observation line, the current on this line will be a travelling wave. Using the Green's functions for the substrate we can calculate the field that this current distribution will cause on the observation line. Because this line is perfectly matched at both ends, it can be regarded as a section of an infinitely long line. The response of such an infinite line to a incident spatial Dirac field impulse at position $\mathrm{x}=0$ will be equal to:

$$
I_{\text {pulse }}(x)=A e^{-\gamma|x|}+I_{\text {pulse }}^{\text {ho }}(x)
$$

$\mathrm{I}^{\text {ho }}$ pulse are the higher order modes. These will only propagate over a very short distance and are therefore ignored. If we look at the Dirac impulse as a one-volt source and replace the two semi-infinite pieces of line with two impedances of $Z_{c}$ ohms then it is easy to see that the amplitude $A$ in (1) will be equal to $1 /\left(2 Z_{c}\right)$. We can now find the current (from which the S-parameters can be calculated) from a convolution of the impulse response of the line (1) and the incident field on the line $\left(E_{2}=Z_{21} I_{1}\right)$. For an observation line with $N_{2}$ basisfunctions:

$$
I_{2}(n)=\sum_{i=1}^{N_{2}} I_{p u l s e}(n-i) E_{2}(i)
$$




\section{INTRA-DISCONTINUITY COUPLING}

This second module will calculate the couplings between the discontinuities of the circuit. If these discontinuities are small compared to the wavelength and the distance between them is big compared to their size then their radiation behavior is comparable to that of a dipole. In this section we will discuss how dipoles can be used to calculate the coupling between the circuit's discontinuities in a fast, approximate, way. The better the two above-mentioned assumptions are met, the better the approximation will be. The approximation can always be improved by using more dipoles in the component's model. Because we only calculating first order coupling again (ignoring the influence on of components in the vicinity on this component's currents) we can store the dipole excitations and positions in a library system for each component. This new method uses far less unknowns than the method of moments.

Data are added to the S-parameter black box model of the component. This data describes the relation between the incoming (port) waves and the currents on the dipoles

$$
\left[\begin{array}{l}
i_{1} \\
\vdots \\
i_{M}
\end{array}\right]=\left[\begin{array}{llc}
t_{11} & \cdots & t_{1 N} \\
\vdots & \ddots & \vdots \\
t_{M 1} & \cdots & t_{M N}
\end{array}\right]\left[\begin{array}{l}
v_{1}^{+} \\
\vdots \\
v_{N}^{+}
\end{array}\right]
$$

and the relation between incident fields on the dipoles and outgoing waves:

$$
\left[\begin{array}{l}
v_{1}{ }^{-} \\
\vdots \\
v_{N}{ }^{-}
\end{array}\right]=\left[\begin{array}{llc}
r_{11} & \cdots & r_{1 M} \\
\vdots & \ddots & \vdots \\
r_{N 1} & \cdots & r_{N M}
\end{array}\right]\left[\begin{array}{l}
e_{1} \\
\vdots \\
e_{M}
\end{array}\right]
$$

The new, extended description of the discontinuity becomes:

$$
\left[\begin{array}{l}
V^{-} \\
I
\end{array}\right]=\left[\begin{array}{ll}
S & R \\
T & X
\end{array}\right]\left[\begin{array}{l}
V^{+} \\
E
\end{array}\right]
$$

In which $\mathrm{S}$ is the S-parameter matrix, and $\mathrm{R}$ and $\mathrm{T}$ are the matrices defined in (3) and (4). The $\mathrm{X}$ submatrix describes the reflections of incident fields on the component. Because of these reflections, indirect paths can be formed between 2 discontinuities through other discontinuities. The additional fields that are caused by these indirect paths are small compared to the direct field. Therefore the $\mathrm{X}$ submatrix is neglected and thus set to zero.

Using the new description we can now combine all the S-matrices of the discontinuities into one big Smatrix, which includes mutual coupling between them:

$$
\left[\begin{array}{l}
V_{1}^{-} \\
\vdots \\
\vdots \\
V_{D}^{-}
\end{array}\right]=\left[\begin{array}{cccccc}
S_{1} & R_{1} G_{1,2} T_{2} & R_{1} G_{1,3} T_{3} & \cdots & R_{1} G_{1, D} T_{D} \\
& & & & & \\
R_{2} G_{2,1} T_{1} & S_{2} & R_{2} G_{2,3} T_{3} & \ldots & R_{2} G_{2, D} T_{D} \\
\vdots & & \ddots & & \\
& & & & & \\
R_{D} G_{D, 1} T_{1} & \cdots & & R_{D} G_{D, D-1} T_{D-1} & S_{D}
\end{array}\right]\left[\begin{array}{l}
V_{1}^{+} \\
\vdots \\
\\
\vdots \\
V_{D}^{+}
\end{array}\right]
$$

Where $G_{i, j}$ is a matrix that relates the field on i's dipoles to the current on $j$ 's. The matrix (6) is named $S_{d}$. It describes all the discontinuities and their mutual interaction through radiation as a single big S-port. By combining it with the line's S-parameters we can eliminate all waves on internal ports. What remains are the S-parameters between externally fed ports.

The optimal dipole positions are found through the use of an optimisation routine. They don't change much as a function of frequency. If the discontinuity has $\mathrm{N}$ ports than excitation of each of these ports will generate a distinct field distribution. The optimisation routine tries to position and feed the dipoles in such a way that, at the centre frequency, their total field resembles these field distributions as much as possible in a number of 
well chosen testpoints. Only the positions of the dipoles are variables for the optimisation: The optimal dipole excitations for a certain position can be found using a least square method.

The T-matrix (the dipole excitations for each port) is calculated for each frequency after the ideal dipole positions have been calculated for the mid-band frequency. This is done using the least square method again. The R matrix can be calculated from the T-matrix through reciprocity. It can be proved that $\mathrm{R}=\mathrm{T} / 2$.

\section{NUMERICAL RESULTS}

The modules of the method are first tested separately and the relation between the approximation error and the proximity of the components is investigated. Then a simple circuit is analysed using both the modules and the S-parameters are compared to those calculated with the Method of Moments.

To find the minimum distance at which the line coupling method still yields accurate results the coupling between a pair of parallel lines is calculated for different distances. Both horizontal lines have a length of 18 $\mathrm{mm}$ and a width of $.61 \mathrm{~mm}(50 \mathrm{ohm})$. They start at the same X-position. The substrate is $0.635 \mathrm{~mm}$ thick and $\varepsilon_{\mathrm{r}}=9.9$. The coupling was calculated and compared to the standard method of moments (using $80 \times 5$ segments for both lines) for three distances: $3 \mathrm{~mm}, 1.5 \mathrm{~mm}$ and $1 \mathrm{~mm}$. The continuous line in figure 1 is the result using the standard method of moments, the dot-dash line the new method. Ports 1 and 2 are on the observation line, ports 3 and 4 on the source line. Ports 1 and 3 are on the left, ports 2 and 4 on the right. The results are usable up to $1.5 \mathrm{~mm}$. The maximum coupling is then $-10 \mathrm{~dB}$.

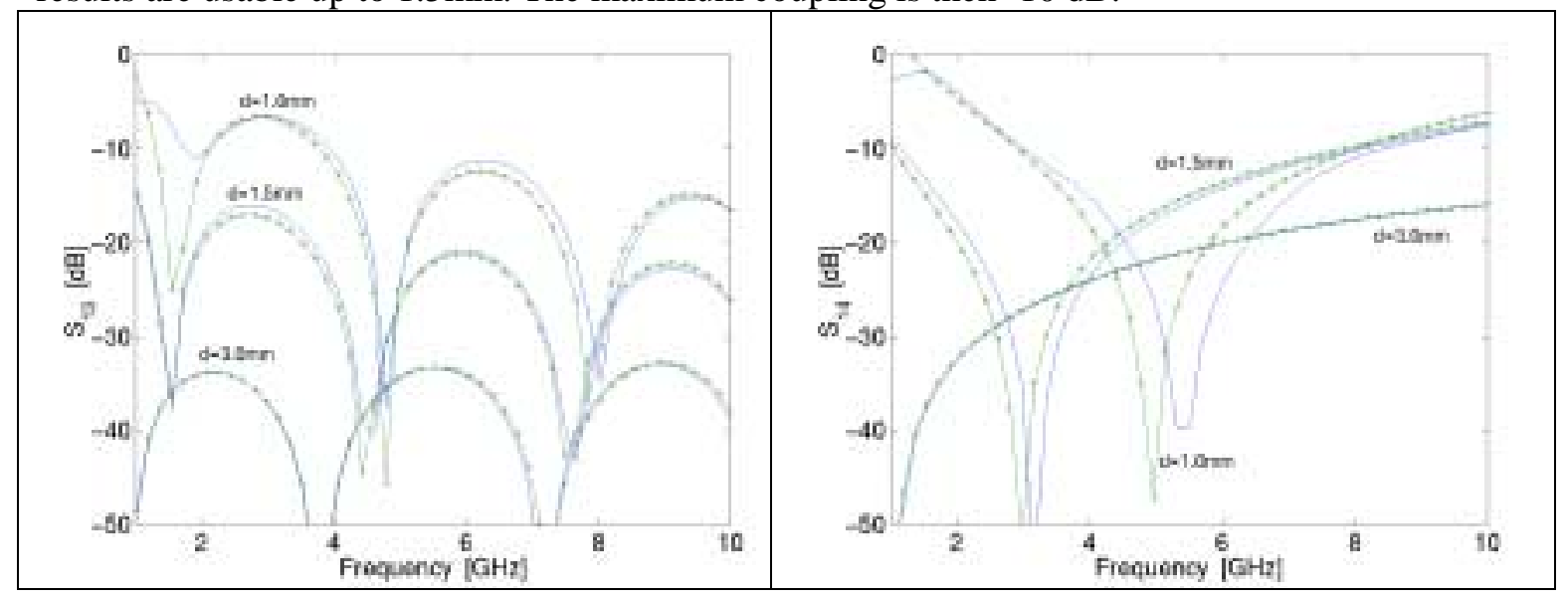

Figure 1. Comparison between M.o.M. and new method for coupling between a parallel pair of lines for distances of $1,1.5$ and $3 \mathrm{~mm}$.

The inter discontinuity coupling module is tested using the T-junction shown in figure 2. A 6 and 3-dipole model is calculated for this discontinuity (with 100 testpoints in a circle with $R_{t}=4 \mathrm{~mm}$ ) and the fields generated by the models are compared to those of the component as a function of frequency and distance. The calculated model is then used to calculate the S-parameters for the 2 coupled T-junctions. The circuit is placed on a substrate with $\varepsilon_{\mathrm{r}}=9.9$ and a thickness of $.631 \mathrm{~mm}$. For this substrate the lines have a characteristic impedance of $50 \mathrm{ohms}$. For the M.o.M. both junctions use 88 rooftop basis functions. In all the 


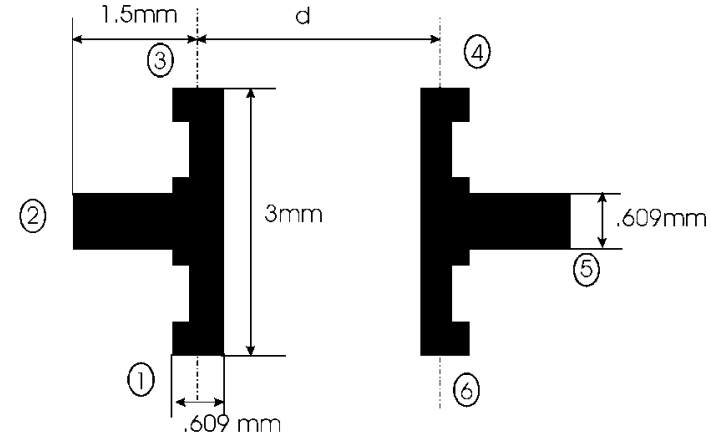

Figure 2. Two T-junctions that will be used to check the inter discontinuity coupling module. Port numbers are indicated following figures the T-junction is positioned with its middle leg pointing down. The dipole model can be verified by analyzing the ratio of the maximum error (over all testpoints) to the maximum field strength. Over a frequency range of 1 to $10 \mathrm{GHz}$, for a testpoint radius of $4 \mathrm{~mm}$, this ratio has a maximum value of $-30 \mathrm{~dB}$ for a 6 dipole and $-15 \mathrm{~dB}$ for a 3 dipole model. If plotted as a function of distance, then the same ratio is below $-30 \mathrm{~dB}$ for the 6 dipole model at distances greater than $3 \mathrm{~mm}$, at $30 \mathrm{GHz}$.

The T-junctions in figure 2 will now be fed by 6 lines. Each line is $9.135 \mathrm{~mm}$ long and $.61 \mathrm{~mm}$ wide $(50 \mathrm{ohm})$ and is segmented for the M.o.M. with a $3 \times 45$ mesh.

The S-parameters for this simple circuit are calculated using the new method and compared to the M.o.M. solution. Figure 3 shows this comparison. The couplings are split up per type: line-line, line-discontinuity, discontinuity-discontinuity, and full coupling. Each graph represents the coupling between two ports. The port numbering is shown in figure 2 .
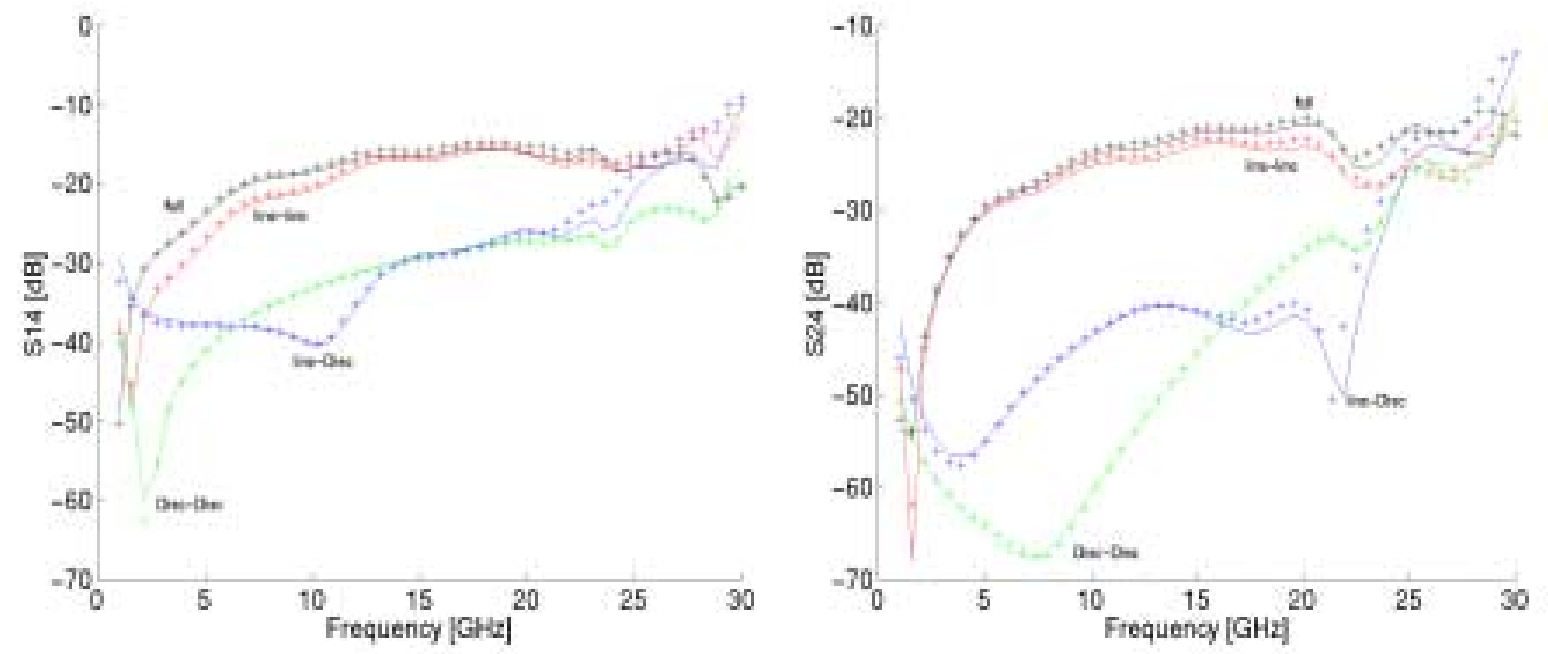

Figure 3. Coupling contributions for T-junctions fed by six lines split up by type. $d=3 \mathrm{~mm}$.

\section{CONCLUSIONS}

The Main advantage of the method is the speed up. The moment method uses 2.142 seconds to set up its matrix, .345 seconds to solve it and 3.427 seconds to deembed the 6 ports. Only $.152 \mathrm{sec}$ are needed for the new method. For large circuits the speed up increases because the inversion time rises proportional to the 3 power of the number of unknowns. The dipole model needs no inversion and will be much faster for large circuits.

Also, the new model needs far less memory than the moment method because only couplings between dipoles of two discontinuities are present in the computers memory.

\section{REFERENCES}

[1] Bart L.A. Van Thielen and Guy A.E. Vandenbosch, Fast transmission line coupling calculation using a convolution technique, IEEE Trans. on Electromagnetic Compatibility, vol EMC-43, no 1, pp. 11-17 Feb. 2001 